\title{
179. Operative Behandlung von Knochenmetastasen des Beckens und des proximalen Femur
}

\author{
W. Mutschler, C. Burri und C. Etter \\ Klinik für Unfallchirurgie der Universität Ulm, Steinhövelstraße 9, D-7900 Ulm
}

\section{Operative Treatment in Metastases of the Pelvis and Proximal Femur}

Summary. Metastases of the pelvis and proximal femur are treated by total hip prosthesis or a combination of bone cement and metal implants. In this way, in 80 of 81 patients the lower extremity could be conserved, and 73 patients were able to walk. Of the 17 local complications, e.g., luxation of the prosthesis, 14 were curable by reoperation. The mean survival time after operation was 9 months.

Key words: Pelvis/femur metastases - Operation results.

Zusammenfassung. Die Verbundosteosynthese und der endoprothetische Ersatz des Hüftgèlenkes sind als funktionserhaltende Eingriffe die Methode der Wahl bei Metastasen im Becken und proximalen Femur. Bei 81 Patienten konnte damit in 80 Fällen die untere Extremität erhalten werden. 73 Patienten erlangten wieder Gehfähigkeit. Von 17 lokalen Komplikationen wurden 14 durch einen Reeingriff behoben. Die durchschnittliche Überlebenszeit betrug 9 Monate postoperativ.

Schlïsselwörter: Becken/Femur-Metastasen - Operationsergebnisse.

\section{Die Behandlung ossärer Metastasen durch gelenkprothetischen Ersatz und Verbundosteosynthese}

\author{
F. Schmülling, K. P. Schmit-Neuerburg und G. Roggenland \\ Universitätsklinikum der Gesamthochschule Essen, Abteilung für Unfallchirurgie, \\ Hufelandstraße 55, D-4300 Essen
}

\section{Treatment of Bone Metastases by Alloarthroplasty and Combination Osteosynthesis}

\begin{abstract}
Summary. The indication for stabilization of pathologic or impending fractures caused by bone metastases is clearly defined : depending on the number and seat of the metastases, the hipjoint or humerus head need to be replaced by an isoelastic or tumor prosthesis, whereas metastases of the shaft are treated by combination osteosynthesis with plate or intramedullary nail and bone cement. This method, filling the intramedullary cavity with a nail $2-3 \mathrm{~mm}$ thinner than usual and bone cement, seems to guarantee more stability in case the osteolysis proceeds to the shaft.
\end{abstract}

Key words: Bone metastases - Prosthetic replacement - Combination osteosynthesis.

Zusammenfassung. Die Indikation zur Stabilisierung drohender oder pathologischer Frakturen infolge Knochenmetastasen ist klar definiert: Abhängig von Zahl und Sitz der Metastasen werden der Oberarmkopf und das Hüftgelenk durch isoelastische oder Tumorprothese ersetzt, während Schaftmetastasen durch Verbundosteosynthese mit Platte oder Marknagel versorgt werden. Die letztere Methode, bei der die Markhöhle mit einem 2-3 mm dünneren Marknagel als gewöhnlich zusammen mit Knochenzement aufgefüllt wird, garantiert u. E. bei fortschreitender Metastasierung ein höheres Maß an Stabilität.

Schliisselwörter: Knochenmetastasen - Endoprothesen - Verbundosteosynthese. 